DOI: $10.3901 / J M E .2020 .17 .190$

\title{
蓝宝石精密磨削表层热修复特性研究*
}

\author{
梁志强 ${ }^{1}$ 钟 劲 ${ }^{2}$ 林 海 $^{2}$ 王银惠 $^{2}$ 奕晓圣 ${ }^{2}$ 王西彬 ${ }^{1}$ \\ 杨海成 ${ }^{3}$ 苏 瑛 $^{3}$
}

(1. 北京理工大学先进加工技术国防重点学科实验室 北京 100081 ;

2. 北京理工大学机械与车辆学院 北京 100081 ;

3. 西安应用光学研究所 西安 710100)

\begin{abstract}
摘要: 蓝宝石精密磨削过程中易产生表面裂纹、微观断裂、位错等缺陷, 同时也会产生残余拉应力, 对后续加工及性能发挥 产生不良影响。采用热修复方法对磨削后蓝宝石表层进行处理, 以氩气作为保护气，在不同热修复温度、保温时间以及降温 速度等条件下, 对 A、C、R、M 面的蓝宝石晶体进行热修复试验。结果表明, 热修复方法能够有效降低表面残余拉应力, 同时提升表面晶格完整性, 其中热修复温度 $1500{ }^{\circ} \mathrm{C}$ 时残余拉应力降幅最高, 可达 $53 \%$; 随着热修复温度的增加以及热修复 时间的增长, 蓝宝石磨削表面残余应力逐渐降低, 而且表面晶格完整性变好; 随着降温速度增加, 表面残余拉应力的降幅减 小，热修复效果减弱；在不同晶向的蓝宝石中， $\mathrm{R}$ 面蓝宝石热修复后表面残余拉应力降幅最大; 通过对抛光后蓝宝石进行热 修复，观察到了表层原子重新排列的台阶结构。证实热修复方法为蓝宝石的低损伤加工提供了一种有效方法。
\end{abstract}

关键词: 蓝宝石; 磨削; 热修复特性; 表层性能; 残余应力

中图分类号: TG156

\section{Research on Thermal Repairing Characteristics of Sapphire Precision Grinding Surface}

\author{
LIANG Zhiqiang ${ }^{1}$ ZHONG Jin ${ }^{2}$ LIN Hai ${ }^{2}$ WANG Yinhui ${ }^{2}$ LUAN Xiaosheng ${ }^{2}$ \\ WANG Xibin $^{1}$ YANG Haicheng ${ }^{3}$ SU Ying $^{3}$
}

(1. Key Laboratory of Fundamental Science for Advanced Machining,

Beijing Institute of Technology, Beijing 100081;

2. School of Mechanical Engineering, Beijing Institute of Technology, Beijing 100081;

3. Xi'an Institute of Applied Optics, Xi'an 710100)

\begin{abstract}
During the precision grinding of sapphire, some defects such as cracks, micro fractures, dislocations are prone to generate on the surface, and residual tensile stress will occur on the surface, this has adverse effects on subsequent proces sing and performance. In this study, thermal repairing method is used to repair the grinded sapphire surface. Under the conditions of different thermal repairing temperature, holding time and cooling rate, thermal repairing test is carried out on sapphire A-plane, C-plane, M-plane and R-plane using argon gas as shielding gas. Experimental results show that thermal repairing can effectively reduce the surface residual tensile stress, improve the surface lattice integrity, and the largest residual stress reduction rate can reach $53 \%$ when the thermal repairing temperature is $1500^{\circ} \mathrm{C}$. With the increase of thermal repairing temperature and holding time, the residual tensile stress of sapphire grinding surface decreases and the surface lattice integrity is improved. With the in crease of cooling rate, the reduction rate of surface residual tensile stress decreases, and the thermal repairing effect is weakened. Among sapphire with different crystal orientations, R-plane sapphire surface has the largest reduction rate of residual stress after thermal repairing. In thermal repairing of polished sapphires, the atomic step morphology after surface atoms rearrangement is observed. Thermal repairing is proved to be an effective method of low damage processing of sapphire.
\end{abstract}

Key words: grinding; sapphire; thermal repairing characteristics; surface properties; residual stress

* 国家自然科学基金(51975053), 国家重点研发计划(2019YFB13111D0), 国 家重点基础研究计划(2015CB059900)和基础科研(JCKY20162088006)资 助项目。20190926 收到初稿, 20191219 收到修改稿 


\section{0 前言}

蓝宝石单晶不仅具有良好的光传播性能、极高 的耐热性以及抗腐蚀性, 还有较好的电气特性以及 介电特性 ${ }^{[1]}$ 。这些化学、光学、物理等方面的优异 性能使蓝宝石晶体被广泛应用于光学电子、航空航 天、照明等领域 ${ }^{[2]}$ 。尤其是在一些关键应用领域, 需要大量高品质低损伤的蓝宝石晶体元件, 这对蓝 宝石晶体的精密与超精密加工工艺提出了严格要求。 采用金刚石砂轮对蓝宝石晶体进行超精密磨削, 其 材料去除率较高, 并能获得较高的精度; 但是蓝宝 石单晶磨削表层会不可避免地产生表面与亚表面裂 纹、变质层、位错等缺陷, 这些加工缺陷的存在增 加了后续光整加工的难度和时间 ${ }^{[3]}$ 。为实现蓝宝石 超光滑低损伤高效加工, 采用热修复的方法对超精 密磨削蓝宝石表面进行一定的改善, 缩减后续研磨 抛光的时间, 提高加工效率。

近年来, 随着蓝宝石的广泛应用, 许多学者为 改善蓝宝石超精密加工表面质量进行一些热处理实 验研究。张伟 ${ }^{[4]}$ 研究了不同热处理工艺参数对 $\mathrm{C}$ 向 蓝宝石抛光基片表面形貌的影响规律, 发现 $900{ }^{\circ} \mathrm{C}$ 以上退火时, 蓝宝石基片出现清哳的台阶结构; YATSUI 等 ${ }^{[5]}$ 研究了回火处理对 $\mathrm{C}$ 面蓝宝石祄底表 面形态的影响, 发现在退火过程中引入回火, 成功 获得了蓝宝石原子台阶结构; ZHAO 等 ${ }^{[6]}$ 在氮气气 氛下研究了蓝宝石祄底上不同厚度的溅射 AIN 薄膜 的热处理工艺, 研究结果表明在 $1400 \sim 1700{ }^{\circ} \mathrm{C}$ 热 处理后, 这些 AlN 膜的晶体质量显着提高, 指出晶 体质量改善与高温下的固相反应有关; PINKAS 等 ${ }^{[7]}$ 研究了不同的热处理条件对梯度法生长 $\mathrm{A}$ 向和 $\mathrm{C}$ 向 抛光后蓝宝石基片的表面形貌以及亚表面损伤层厚 度的影响, 研究结果表明, 在 $1450{ }^{\circ} \mathrm{C}$ 空气气氛下 热处理 $72 \mathrm{~h}$ 后抛光蓝宝石表面形成宽的台阶结构, 蓝宝石表面损伤得到了很大程度上热愈合; CUCCUREDDU 等 ${ }^{[8]}$ 研究了 $\mathrm{C}$ 向蓝宝石抛光基片在 不同的热处理条件下表面形态的变化, 研究结果表 明在空气气氛下经过 $1100{ }^{\circ} \mathrm{C}$ 热处理 $12 \mathrm{~h}$ 后 $\mathrm{C}$ 面 蓝宝石表面出现规则的台阶和台面结构, 但是台面 的边缘不是很清晰, 平均台阶高度为 $(1.8 \pm 0.7) \mathrm{nm}$, 平均平台宽度为 $(43.6 \pm 14.7) \mathrm{nm}$ 。针对蓝宝石磨削的 表面损伤和表面残余应力等缺陷, 提出蓝宝石磨削 表面的热修复方法。开展蓝宝石磨削表层热修复试 验, 基于蓝宝石晶体结构特性和滑移特性分析蓝宝 石精密磨削表层性能热修复机理, 揭示蓝宝石磨削 表面晶格完整性和表面残余应力热修复特性。

\section{1 蓝宝石精密磨削表层热修复原理}

\section{1 蓝宝石单晶晶体结构特性}

蓝宝石单晶 $\left(\alpha-\mathrm{Al}_{2} \mathrm{O}_{3}\right)$ 点阵由 $\mathrm{Al}^{3+}$ 和 $\mathrm{O}^{2-}$ 构成, 根 据蓝宝石单晶结构中铝离子和氧离子之间距离及其 三方晶系晶格特性，其晶体结构以及局部放大示意 图如图 1 所示, 图中小圆代表铝离子，大圆代表氧 离子, 不同晶面的铝离子、氧离子密度是不同的, 其间形成的是离子键, 其排列是以六方最紧密堆积 的方式。从局部示意图可以看出一个铝离子连接六 个氧离子, 铝离子距离上下两层氧离子的距离是不 同的, 在 $C$ 轴方向上, 距离较大的三组 $\mathrm{Al}-\mathrm{O}$ 的间 距为 $0.197 \mathrm{~nm}$ 、另外三组 Al-O 的间距为 $0.187 \mathrm{~nm}$, 当温度升高时晶体结构的键长会变大, 铝离子的位 置相对氧离子的位置来说改变比较大, 使得表面原 子重新排列, 同时密排六方点阵结构变得更加有序, 进而使得表面晶格完整性更好 ${ }^{[9]}$ 。
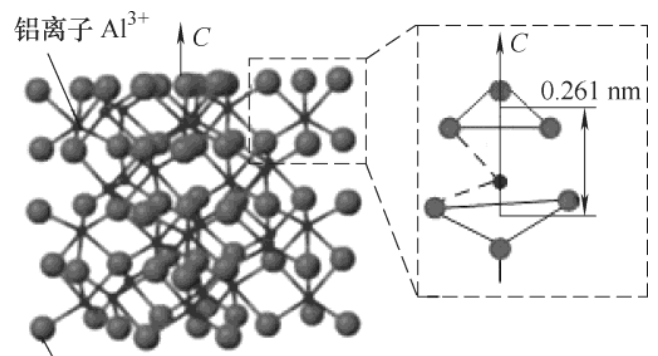

氧离子 $\mathrm{O}^{2}$

图 1 蓝宝石单晶结构示意图

\section{2 蓝宝石单晶晶体滑移特性}

蓝宝石晶体共有 3 种滑移系如表 $1^{[9]}$ 所示, 其 中棱面和基面两种滑移系类型如图 $2^{[9]}$ 所示, 最易滑 移的面是 $\mathrm{C}(0001)$ 面, 以沿 $(11 \overline{2} 0)$ 方向为主导底面滑 移 ${ }^{[9]}$ 。由于热修复过程中的温度场是非线性的, 晶 体的塑性变形主要通过滑移实现, 温度对蓝宝石中位 错有一定的影响, 在 $300 \sim 900{ }^{\circ} \mathrm{C}$ 的温度范围内, 沿 着棱面滑移相对容易的局部滑移被观察到, 在温度高 于 700 900 ${ }^{\circ} \mathrm{C}$, 蓝宝石中发现底部的位错滑移 ${ }^{[9]}$ 。

底面滑移的激活能取决于温度和相应的载荷, 在磨削加工过程中蓝宝石晶体某一晶面如果受到比 蓝宝石的屈服强度高的切应力时, 晶面就会出现滑 移的现象，晶体一般是沿着原子最密集分布的方向 发生滑移, 发生滑移晶体表面原子密度较大。位错 是晶体中局部滑移区域的边界线, 即晶体中的一种 线缺陷。发生位错的部位原子发生严重错排, 蓝宝 石晶体的晶格相对于无位错处发生位置改变。材料 应力与位错缺陷的产生有直接关系, 样件位错密度 的大小可以一定程度上反应晶体内部应力的大 
小 ${ }^{[10]}$ 。在热修复过程中由于温度的升高, 在蓝宝石 晶体内部原子重新排列, 特别是在位错区域, 晶体 结构变得更加有序, 单个位错缺陷处所具有的非平 衡态能量下降, 同时位错面积减小和位错密度下降, 从而造成残余应力减小 ${ }^{[11]}$; 缺陷亚表面层结构也发 生变化, 出现再结晶现象, 有利于局部残余应力的 释放, 造成位错密度降低和残余应力急剧减小 ${ }^{[9]}$ 。

表 1 蓝宝石中可能的滑移系

\begin{tabular}{lccc}
\hline \multicolumn{1}{c}{ 滑移系类型 } & 滑移面 & 滑移方向 & 等效系数 \\
\hline 基面 1 & $\mathrm{C}$ 面 $(0001)$ & $<1 \overline{2} 10>$ & 3 \\
基面 2 & $\mathrm{C}$ 面 $(0001)$ & $<10 \overline{1} 0>$ & 3 \\
棱柱面 1 & $\mathrm{~A}$ 面 $(1 \overline{2} 10)$ & $<10 \overline{1} 0>$ & 3 \\
棱柱面 2 & $\mathrm{M}$ 面 $(10 \overline{1} 0)$ & $<1 \overline{2} 10>$ & 3 \\
棱柱面斜方向滑移 & $\mathrm{A}$ 面 $(1 \overline{2} 10)$ & $<10 \overline{1} 2>$ & 6 \\
菱形面 & $\mathrm{R}$ 面 $\{10 \overline{1} 1\}$ & $<10 \overline{1} 2>$ & 3 \\
\hline
\end{tabular}

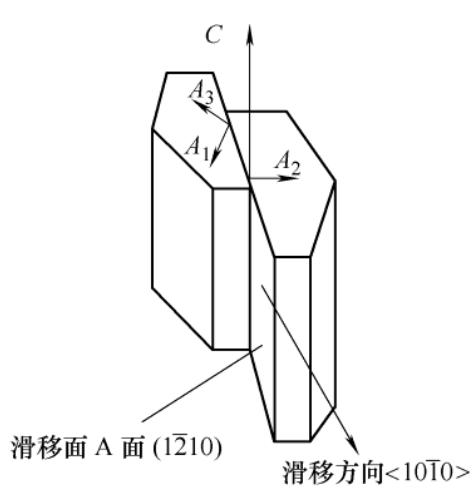

(a) 棱面滑移系

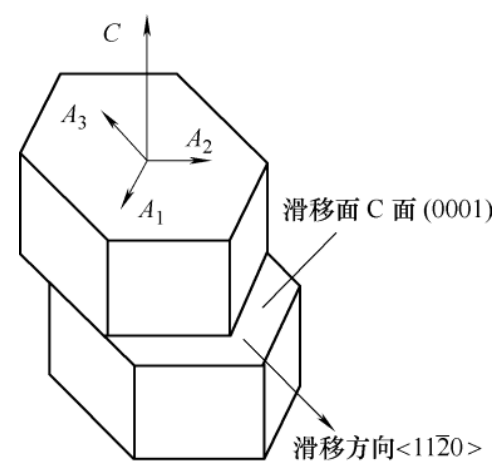

(b) 基面滑移系

图 2 蓝宝石棱面和基面滑移系的示意图

\section{2 蓝宝石精密磨削表层热修复试验}

\section{1 试验条件}

试验材料为 $\mathrm{C}$ 面蓝宝石晶块, 尺寸为 $12 \mathrm{~mm} \times$ $10 \mathrm{~mm} \times 6 \mathrm{~mm}$ 。磨削试验在 NAS520X-CNC 长岛精 密磨床上进行, 如图 3 所示, 采用树脂结合剂金刚 石砂轮对蓝宝石单晶进行磨削加工, 为保证良好的
冷却效果，采用逆磨湿式的方法加工，蓝宝石磨削 的砂轮及磨削工艺参数见表 2 , 每次磨削前用白刚 玉油石进行修整以保证试验条件的一致性。

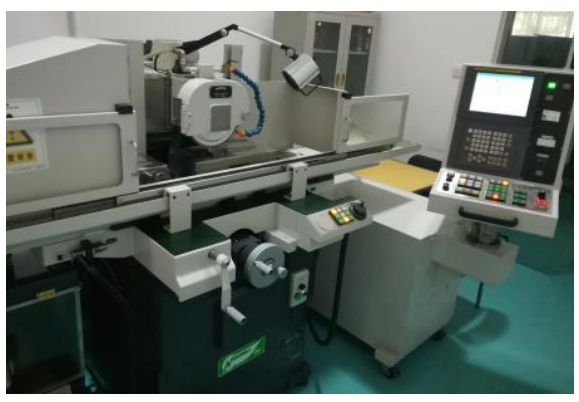

图 3 磨削试验装置

表 2 蓝宝石单晶磨削砂轮及磨削工艺参数

\begin{tabular}{lc}
\hline \multicolumn{1}{c}{ 工艺参数 } & 具体参数 \\
\hline 砂轮 & 1500\# SD, 14A1 250D-3X-15U-50.8H-25T \\
冷却液 & 尤希路 SC-25AC 水基冷却液 \\
工件 & C 面蓝宝石 \\
磨削深度 $a_{\mathrm{p}} / \mu \mathrm{m}$ & 1 \\
砂轮速度 $v_{\mathrm{s}} /(\mathrm{m} / \mathrm{s})$ & 10.5 \\
进给速度 $v_{\mathrm{f}} /(\mathrm{m} / \mathrm{min})$ & 4 \\
\hline
\end{tabular}

蓝宝石磨削表面的热修复采用 SK-G06163 型 气体管式炉, 热修复温度分别为 $800{ }^{\circ} \mathrm{C} 、 1100{ }^{\circ} \mathrm{C}$ 、 $1300{ }^{\circ} \mathrm{C} 、 1500{ }^{\circ} \mathrm{C}$, 热修复时间分别为 $1 \mathrm{~h} 、 3 \mathrm{~h}$ 、 $5 \mathrm{~h} 、 7 \mathrm{~h}$, 平均升温速度均为 $220{ }^{\circ} \mathrm{C} / \mathrm{h}$, 平均降温 速度为 $140{ }^{\circ} \mathrm{C} / \mathrm{h}$, 选择氩气作为保护气。降温速度 是热修复过程比较重要的工艺参数, 合理的设定降温 速度有利于获得表面低应力的晶体。加热到给定温度 保温一定时间后开始降温, 在降温开始阶段蓝宝石外 表面温度下降较快, 此时工件内部的温度仍比较高。 如果降温速度过快, 晶体外表层和内部的横向温度梯 度将会变大，导致残余拉应力的产生 ${ }^{[12]}$, 产生不利 影响。

在研究降温速度对蓝宝石磨削表层晶格完整性 的影响试验中，将工件升温到 $1100{ }^{\circ} \mathrm{C}$ 后，采用不 同的降温速度进行热修复。为了得到更好的蓝宝石 表面质量, 分温度区间控制降温速度, $500{ }^{\circ} \mathrm{C}$ 以后 随炉冷却。选择四个不同的降温速度如表 3 所示。

表 3 降温速度表

\begin{tabular}{|c|c|c|c|c|}
\hline & \multicolumn{4}{|c|}{ 速度/( $\left.{ }^{\circ} \mathrm{C} / \mathrm{h}\right)$} \\
\hline & $1100 \sim 1000{ }^{\circ} \mathrm{C}$ & $1000 \sim 800{ }^{\circ} \mathrm{C}$ & $800 \sim 500{ }^{\circ} \mathrm{C}$ & 平均速度 $/\left({ }^{\circ} \mathrm{C} / \mathrm{h}\right)$ \\
\hline 1 & 60 & 150 & 300 & 140 \\
\hline 2 & 80 & 180 & 350 & 180 \\
\hline 3 & 100 & 210 & 400 & 220 \\
\hline 4 & 120 & 240 & 450 & 260 \\
\hline
\end{tabular}




\section{2 检测方法}

选用图 4 所示的 D8-DISCOVER 型高分辨 X 射 线衍射仪对蓝宝石精密磨削表层性能进行测量。该 $\mathrm{X}$ 射线衍射仪 theta、2theta 轴最小步距 $0.0001^{\circ}, \mathrm{X}$ 射线源为 $\mathrm{Cu}$ 的 $\mathrm{K}_{\alpha}$ 辐射, 波长为 $\lambda=0.15418 \mathrm{~nm}$, 测量过程中采用 $\omega$-scan 的扫描方式。

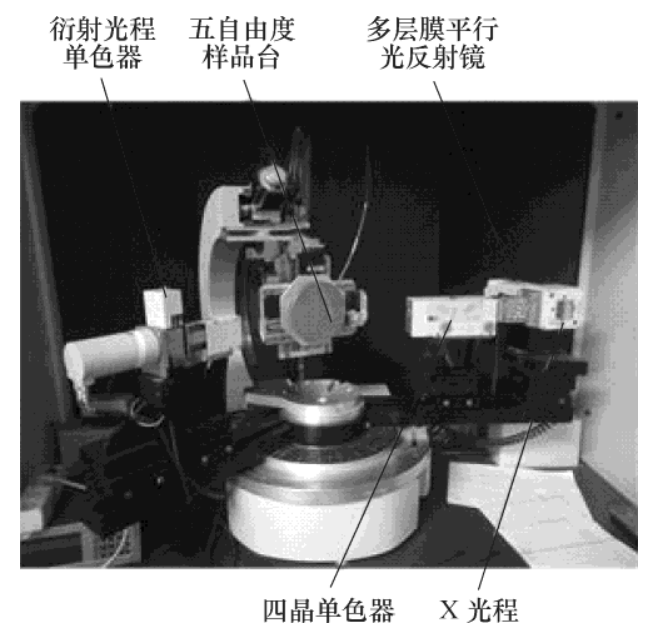

图 4 高分辨 $\mathrm{X}$ 射线衍射仪

通过 X 射线衍射仪测量出蓝宝石加工表面摇摆 曲线示意图如图 5 所示, 测得的摇摆曲线的对称性, 可以直观的反应出蓝宝石的成分和结构是否均匀, 如果晶体内部具有相对均匀的成分及结构, 则测得 的摇摆曲线对称性较好。在摇摆曲线衍射最大强度 的一半处, 作与背底平行的弦, 如图 5 所示, 用此弦 长来表示衍射线的半高宽(Full width at half maximum, $\mathrm{FWHM}$ ), 记做 $\beta$, 可以由式(1)计算出具体数值。摇 摆曲线半高宽的主要影响因素是材料内部位错、晶 体结构均匀性等, 所以 FWHM 值的大小可以直接 反应出蓝宝石表面晶格完整性的好坏, FWHM 值越 小说明晶体表面晶格完整性越好, FWHM 值越大说 明其晶格完整性越差。

$$
\beta=\Delta 2 \theta=2 \theta_{2}-2 \theta_{1}
$$

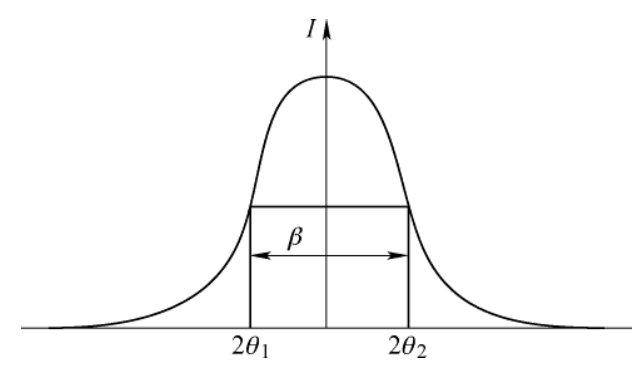

图 5 摇摆曲线示意图

蓝宝石表面应变 $\varepsilon$ 与半高宽值之间满足如下 公式 ${ }^{[7]}$

$$
\varepsilon=\frac{\beta \cdot \tan \theta_{0}}{4}
$$

式中, $\beta$ 是摇摆曲线半高宽值; $\theta_{0}$ 是获得摇摆曲线 时入射 $X$ 射线与蓝宝石表面的夹角; 根据蓝宝石的 弹性模量 $E$, 其加工表面的残余应力可以由式(3)计算

$$
\sigma=\varepsilon E
$$

\section{3 试验结果与讨论}

\section{1 蓝宝石磨削表层晶格完整性的热修复特性}

(1) 热修复温度对蓝宝石磨削表层晶格完整性 的影响

$\mathrm{C}$ 面蓝宝石经过 $800{ }^{\circ} \mathrm{C} 、 1100{ }^{\circ} \mathrm{C} 、 1300{ }^{\circ} \mathrm{C}$ 、 $1500{ }^{\circ} \mathrm{C}$ 热修复 $3 \mathrm{~h}$ 后, 测得不同热修复温度下的 摇摆曲线如图 6 所示, 通过摇摆曲线计算出衍射线 半高宽值随热修复温度的变化规律如图 7 所示。

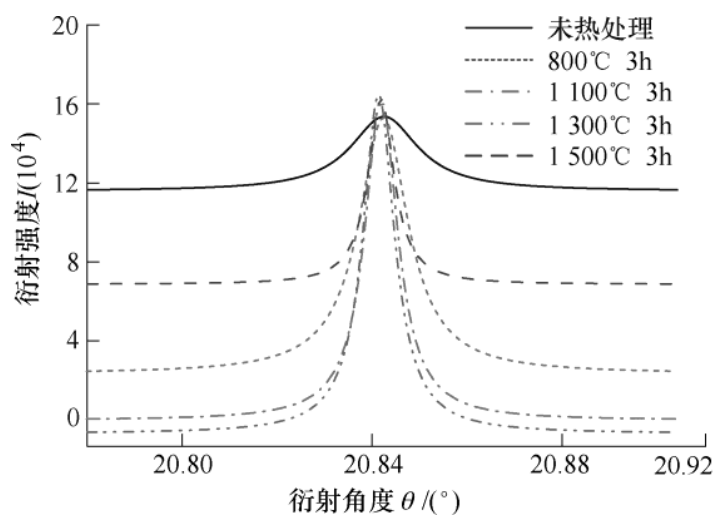

图 6 不同热修复温度下摇摆曲线

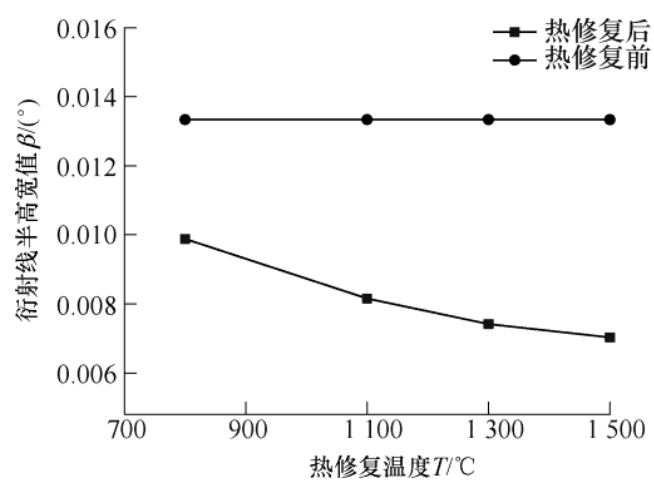

图 7 热修复前后表面半高宽值随热修复温度变化规律

从图 7 中可以看到相对于未热修复蓝宝石, 热 修复后蓝宝石表面衍射线的半高宽值明显降低, 并 且随着热修复温度的升高, 蓝宝石表面衍射线的半 高宽值逐渐降低，说明改变热修复温度可以有效提 高蓝宝石表面晶格完整性, 这是由于温度升高后蓝 宝石表面顶层原子在高温时运动加剧, 更多原子获 得足够的能量后发生迁移, 使得表面原子重新排列, 形成表面自由能更低的结构, 这样的表面结构更稳 定, 从而使得蓝宝石磨削表面晶格完整性更好。 
（2）热修复时间对蓝宝石磨削表层晶格完整性 的影响

C 面蓝宝石经过 $1100{ }^{\circ} \mathrm{C}$ 热修复 $1 \mathrm{~h} 、 3 \mathrm{~h} 、 5 \mathrm{~h}$ 、 $7 \mathrm{~h}$ 后, 通过摇摆曲线计算出衍射线的半高宽值随 保温时间的变化规律如图 8 所示。

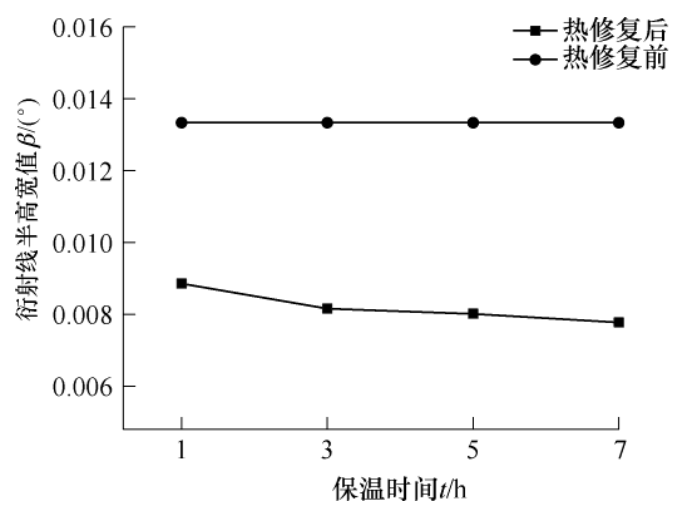

图 8 热修复前后表面半高宽值随保温时间变化规律

从图 8 中可以看出随着保温时间增加蓝宝石表 面半高宽值变化不是很明显, 改变保温时间对半高 宽值影响不显著, 热修复保温时间从 $1 \mathrm{~h}$ 增加到 $3 \mathrm{~h}$ 半高宽值减少 $7.9 \%$, 而从 $3 \mathrm{~h}$ 增加到 $7 \mathrm{~h}$ 半高宽值 仅减少 $4.7 \%$ 。表明保温时间增加, 其表面晶格完整 性没有发生明显变化。

(3) 降温速度对蓝宝石磨削表层晶格完整性的 影响

$\mathrm{C}$ 面蓝宝石在 $1100{ }^{\circ} \mathrm{C}$ 热修复 $1 \mathrm{~h}$, 经过不同 降温速度(表 3)处理后, 通过摇摆曲线计算出衍射线 半高宽值随降温速度的变化规律如图 9 所示。

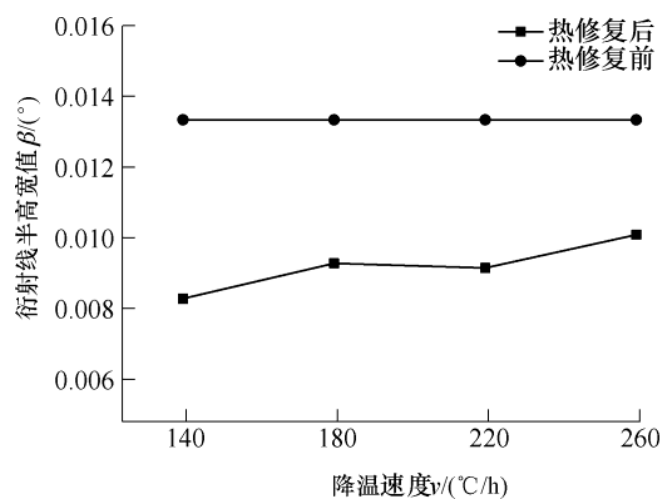

图 9 热修复前后表面半高宽值随降温速度的变化规律

从图 9 中可以看出经过不同降温速度热修复 后蓝宝石表面衍射线半高宽值相对于未经热修 复蓝宝石明显降低, 说明通过改变降温速度可以 有效提高蓝宝石表面晶格完整性, 并且随着降温 速度的提高蓝宝石表面半高宽值逐渐增加, 这是 由于在热修复降温过程中, 蓝宝石晶体外部温度 随之快速下降, 此时晶体内部的温度仍然相对比 较高, 如果采用过快的降温速度, 晶体内部的温
度相对于晶体外表面要高很多, 温度差较大, 从 而一定程度上影响了蓝宝石表面晶格完整性。因 此合理的控制降温速度可以提高蓝宝石表面晶 格完整性。

（4）不同晶向蓝宝石表层晶格完整性热修复特性 蓝宝石材料具有各向异性，具有 $\mathrm{A}$ 面、C 面、 $\mathrm{R}$ 面、 $\mathrm{M}$ 面四个不同晶面，每个晶面上原子密度以 及排布方式都不相同, 图 10 为不同晶面蓝宝石精密 磨削表面在 $1100{ }^{\circ} \mathrm{C}$ 热修复 $1 \mathrm{~h}$ 前后表面晶格完整 性的变化。

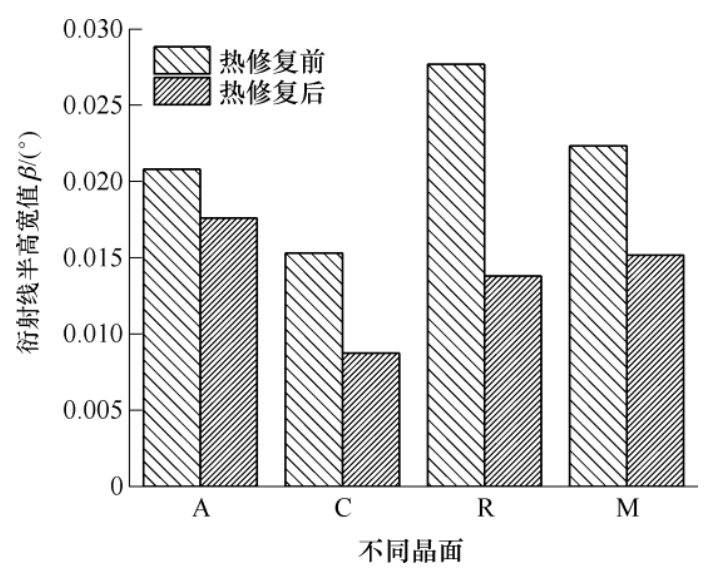

图 10 不同晶面蓝宝石热修复前后表面半高宽值变化

从图 10 中可以看出 $\mathrm{R}$ 面蓝宝石表面半高宽值最 大, 其次是 $\mathrm{M}$ 面、 $\mathrm{A}$ 面, $\mathrm{C}$ 面蓝宝石的半高宽值最小, 说明精密磨削加工后 $\mathrm{C}$ 面蓝宝石表面晶格完整性最好, $\mathrm{R}$ 面蓝宝石表面晶格完整性最差。经过热修复之后不 同晶面蓝宝石表面半高宽值均有所下降，表面晶格完 整都变好，而且 $\mathrm{R}$ 面和 $\mathrm{C}$ 面改善效果明显。

\section{2 蓝宝石表层残余应力的热修复特性}

(1) 热修复温度对蓝宝石表面残余应力的影响。

图 11 为 $\mathrm{C}$ 面蓝宝石经过 $800{ }^{\circ} \mathrm{C} 、 1100{ }^{\circ} \mathrm{C}$ 、 $1300{ }^{\circ} \mathrm{C} 、 1500{ }^{\circ} \mathrm{C}$ 热修复 $3 \mathrm{~h}$ 后, 精密磨削表面残 余应力随热修复温度的变化规律。

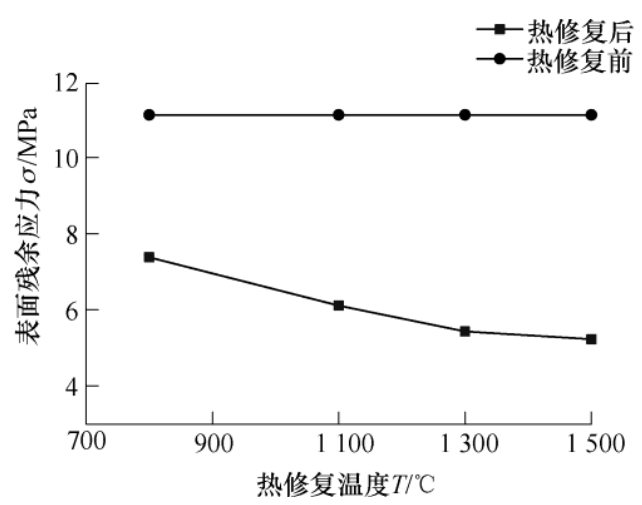

图 11 热修复前后表面残余应力随热修复温度变化规律 从图中可以看出经过不同温度热修复后表面残 
余拉应力相对于未热修复蓝宝石均降低, 并且随着 热修复温度的升高, 表面残余拉应力度逐渐降低, 其中 $1500{ }^{\circ} \mathrm{C}$ 时降幅最大为 53\%。这是因为蓝宝石 磨削加工表层有位错缺陷存在, 而在位错周围由于 原子的错排产生应力场, 随着热修复温度的升高, 蓝宝石磨削表层位错密度降低, 通过位错运动和表 层原子重新排列形成表面自由能较低的结构, 从而 导致表面层中残余应力的松弛, 即残余拉应力下降。

(2) 保温时间对蓝宝石表面残余应力的影响。

图 12 为 C 面蓝宝石经过 $1100{ }^{\circ} \mathrm{C}$ 热修复 $1 \mathrm{~h}$ 、 $3 \mathrm{~h} 、 5 \mathrm{~h} 、 7 \mathrm{~h}$ 后精密磨削表面残余应力随热修复 时间的变化规律。当热修复时间从 $1 \mathrm{~h}$ 增加到 $3 \mathrm{~h}$ 时残余拉应力从 $7.56 \mathrm{MPa}$ 降低至 $6.11 \mathrm{MPa}$, 降低 明显, 但是再继续增加热修复时间, $5 \mathrm{~h}$ 和 $7 \mathrm{~h}$ 热 修复后表面残余拉应力分别为 $6.01 \mathrm{MPa}, 5.82 \mathrm{MPa}$, 降幅没有明显增大, 这是因为 $1100{ }^{\circ} \mathrm{C}$ 的温度提供 蓝宝石表面顶层原子发生迁移重新排列以及位错滑 移所需要的能量有限, 限制残余拉应力进一步降低。

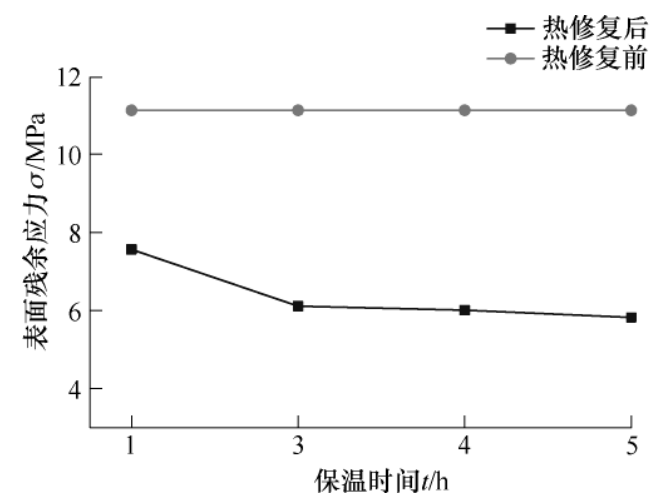

图 12 热修复前后表面残余应力随保温时间变化规律

(3) 降温速度对蓝宝石表面残余应力的影响。

图 13 为 $\mathrm{C}$ 面蓝宝石经过 $1100{ }^{\circ} \mathrm{C}$ 热修复 $1 \mathrm{~h}$ 经过不同降温速度(表 3)处理后精密磨削表面残余 应力的变化规律。

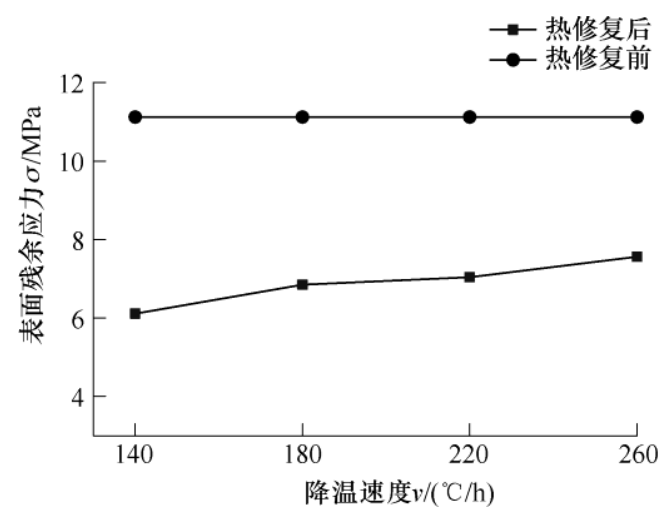

图 13 热修复前后表面残余应力随降温速度变化规律

从图 13 中可以看出经过不同降温速度热修复
后磨削表面残余拉应力相对于未热修复蓝宝石明显 降低, 并且随着降温速度的加快, 表面残余拉应力 降低幅度减小。这是由于降温过程中, 蓝宝石晶体 表面温度快速降低, 而晶体内部温度仍然很高, 晶 体内外温度梯度将变得很大, 使得晶体表层原子重 新排列效果减弱, 热修复效果减弱。

(4) 不同晶向蓝宝石表层残余应力热修复特性。

如图 14 所示为不同晶面蓝宝石精密磨削表面 在 $1100{ }^{\circ} \mathrm{C}$ 热修复 $1 \mathrm{~h}$ 前后表面残余应力的变化。热 修复前 $\mathrm{R}$ 面蓝宝石表面残余应力最小为 $4.61 \mathrm{MPa}$, 然后依次为 $C 、 A 、 M$ 面，热修复后 $A 、 C 、 R 、 M$ 向蓝宝石表面残余应力降幅分别为 $12.8 \%$ 、 $42.4 \%$ 、 $45.6 \% 、 33.6 \%$, R 面蓝宝石表面残余应力降幅最大。

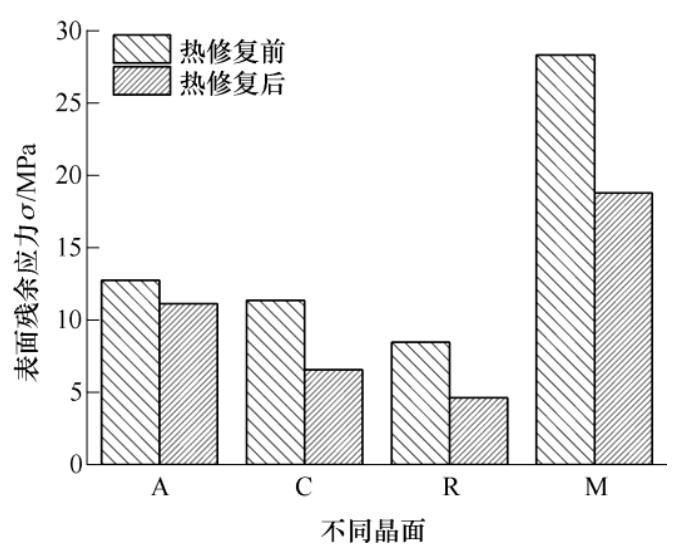

图 14 不同晶向热修复前后残余应力变化

\section{3 蓝宝石热修复过程中的表层形貌变化}

精密磨削后蓝宝石表面粗䊅度在 $10 \sim 100 \mathrm{~nm}$, 直接观察磨削蓝宝石热修复前后表面, 无法观察到 热修复过程中的表层原子迁移现象, 故采用表面粗 粘度为 $0.3 \mathrm{~nm}$ 的抛光后蓝宝石, 在 $1400{ }^{\circ} \mathrm{C}$ 热修复 $3 \mathrm{~h}$ 后, 用原子力显微镜观察热修复前后表面形貌 变化。

蓝宝石单晶的晶格常数 $a=0.476 \mathrm{~nm}, c=1.299 \mathrm{~nm}$, 一个晶胞中含有相间排列铝、氧原子层各 6 层, 相 邻两层氧原子之间的距离为 $c / 6$ 即 $0.216 \mathrm{~nm}$ 。理论 上 $\mathrm{C}$ 面蓝宝石的晶面取向偏差为 $0^{\circ}$, 抛光后可以 获得绝对光滑的表面, 但是在从开始的长晶及定向 处理到最后抛光到成品的加工过程中, 由于线切割 误差, 以及后续磨削、研磨工序的影响, $C$ 轴取向 偏差不再是 $0^{\circ}$, 与理想的 $\mathrm{C}$ 面存在一个错切角 $\theta$ 如图 15 所示。使得铝氧原子层发生了偏转, 进而导 致抛光到原子级光滑后蓝宝石表面会出现的台阶结 构, 且台阶的高度为 $c / 6=0.216 \mathrm{~nm}^{[13]}$ 。

如图 16 所示为抛光处理后蓝宝石祄底表面 AFM 图, 从图中可以看出蓝宝石表面会呈现出波浪 
形的台阶结构, 但是台阶的边缘还不是很清晰, 经 过测量表面主要是由平均高度为 $0.25 \mathrm{~nm}$, 平均宽 度为 $48.63 \mathrm{~nm}$ 的台阶状结构组成, 其高度与蓝宝石 单晶中两个氧原子层之间的距离差不多, 说明当抛 光达到一定程度后就可观察到下图所示由铝原子和 氧原子层层堆叠的台阶形貌。

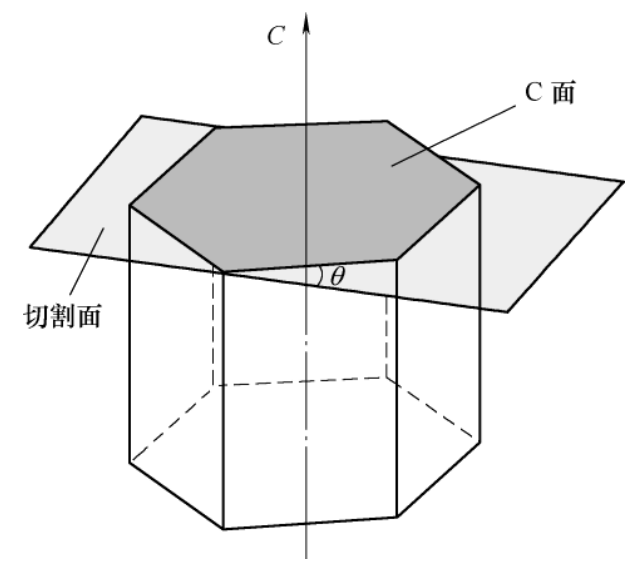

图 15 C 面蓝宝石错切角示意图

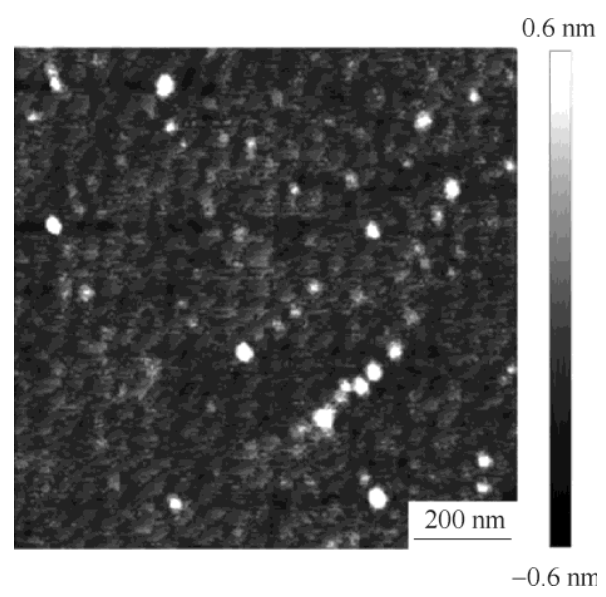

图 16 抛光后蓝宝石表面形貌 $(1 \mu \mathrm{m} \times 1 \mu \mathrm{m})$

如图 17 所示为经过 $1400{ }^{\circ} \mathrm{C}$ 热修复 $3 \mathrm{~h}$ 后蓝宝 石表面形貌图和截面轮廓图, 从图中可以观察到较 宽的、较规则的台阶结构, 经测量表面台阶是由平 均高度为 $1.18 \mathrm{~nm}$, 平均宽度为 $238.62 \mathrm{~nm}$ 的台阶 状结构构成, 是由图 16 中多个小台阶合并之后组成 的, 导致合并后台阶结构宽度和高度增加。这说明 热修复过程蓝宝石表面原子沿着台面和台阶边缘发 生了扩散, 而这种扩散是表面下层的原子不断的向 表面运动, 从而使得台阶不断变宽变高, 在这个过 程中也伴随着蓝宝石内部位错的运动, 即由于热修 复过程中温度上升蓝宝石内部的位错也向自由表面 的运动使得蓝宝石材料从表面下层移动到表层, 并 且这些移动到表层的材料将通过表面扩散达到最低 的能量, 从而导致残余应力下降; 同时在蓝宝石内 部的位错缺陷由于位错的运动, 形成较低能量的结
构, 单个位错缺陷处具有的非平衡态能量降低, 同 时位错面积减小和位错密度下降, 进而使得表面晶 格完整性更好, 残余应力下降。

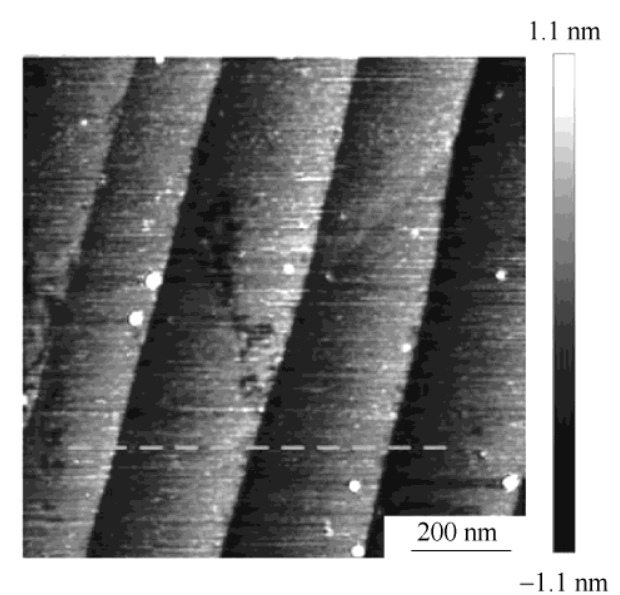

(a) 热修复后蓝宝石表面形貌 $(1 \mu \mathrm{m} \times 1 \mu \mathrm{m})$

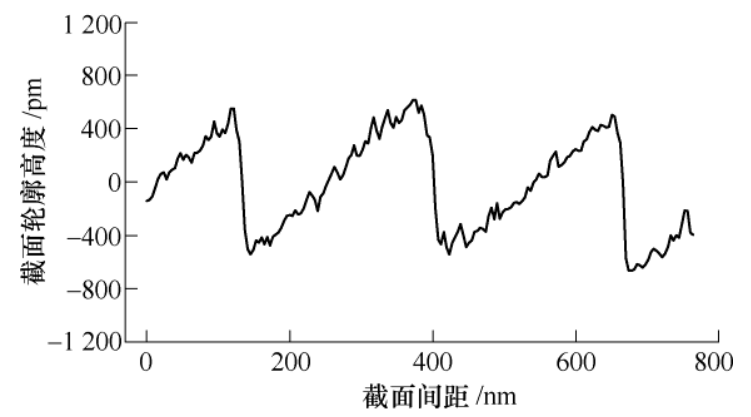

(b) 热修复后蓝宝石表面截面轮廓图

图 17 热修复后蓝宝石表面形貌及截面轮廓图

\section{4 结论}

(1) 对热修复前后蓝宝石磨削加工表面残余应 力进行检测, 揭示了表面残余应力随热修复工艺参 数的变化规律。试验结果表明蓝宝石表面残余拉应 力随着热修复温度和保温时间的增加而减小, 随降 温速度的增加而增加, 其中经过 $1500{ }^{\circ} \mathrm{C}$ 下保温 $3 \mathrm{~h}$ 的热修复, 残余应力降幅最大为 $53 \%$ 。

(2) 通过蓝宝石磨削表面热修复试验, 揭示表 面晶格完整性随热修复工艺参数的变化规律。试验 结果表明蓝宝石表面的晶格完整性随着热修复温度 和保温时间的增加表面晶格完整性越好, 随着降温 速度的增加而修复效果变弱。

(3) 对不同晶向蓝宝石进行热修复试验, 结果表 明, 相同磨削条件加工的不同晶向蓝宝石热修复后 C 面蓝宝石表面晶格完整性最好, $\mathrm{R}$ 面蓝宝石表面残 余拉应力最小为 $4.61 \mathrm{MPa}$, 同时降幅最大为 $45.6 \%$ 。 


\section{参 考 文 献}

[1] NAHAR R K. Study of the performance degradation of thin film aluminum oxide sensor at high humidity[J]. Sensors \& Actuators B Chemical, 2000, 63(1-2): 49-54.

[2] HENDERSON M A. The interaction of water with solid surfaces: Fundamental aspects revisited[J]. Surface Science Reports, 2002, 46(1-8): 1-308.

[3] AKSELROD M S, BRUNI F J. Modern trends in crystal growth and new applications of sapphire $[\mathrm{J}]$. Journal of Crystal Growth, 2012, 360(1): 134-145.

[4] 张伟. 表面处理蓝宝石基片对 $\mathrm{ZnO}$ 薄膜性能的影响[D]. 大连: 大连理工大学, 2013 .

ZHANG Wei. Study of $\mathrm{ZnO}$ films grown on the treated sapphire substrates[D]. Dalian: Dalian University of Technology. 2013

[5] YATSUI T, KURIBARA K, SEKETANI $\mathrm{T}$, et al. Temperature-modulated annealing of c-plane sapphire for long-range-ordered atomic steps[J]. Journal of Physics D: Applied Physics, 2016, 49(11): 115302.

[6] ZHAO Lu, YANG Kun, AI Yujie, et al. Crystal quality improvement of sputtered AlN film on sapphire substrate by high-temperature annealing[J]. Journal of Materials Science: Materials in Electronics, 2018, 29(16): 13766-13773.

[7] PINKAS M, LOTEM H, GOLAN Y, et al. Thermal healing of the sub-surface damage layer in sapphire[J]. Materials Chemistry and Physics, 2010, 124: 323-329.

[8] CUCCUREDDU F, MURPHY S, SHVETS I.V, et al. Surface morphology of c-plane sapphire ( $\alpha$-alumina) produced by high temperature anneal[J]. Surface Science, 2010, 4(10): 10-16.

[9] DOBROVINSKAYA E R, LYTVYNOV L A, PISHCHIK V, Sapphire: Material, manufacturing, applications[M]. New York: Springer, 2013.

[10] 孙瑜. 蓝宝石单晶的位错缺陷及光学性能研究[D]. 天 津：河北工业大学，2013

SUN Yu. Investigation on the dislocations and optical performance of sapphire crystal[D]. Tianjin : Hebei University of Technology, 2013.

[11] 吕汉熊. 蓝宝石生长方法和晶向对晶体质量与性能影 响规律研究[D]. 哈尔滨：哈尔滨工业大学, 2017.

LÜ Hanxiong. Research on influence of sapphire growth process and crystal orientation on crystal quality and performance[D]. Harbin: Harbin Institute of Technology, 2017.

[12] 吴小风. 导模法生长蓝宝石晶体工艺及性能研究 [D]. 南京: 南京航空航天大学, 2015 .

WU Xiaofeng. Technics and performances of sapphire crystals grown by edge-defined film-fed growth (EFG) method[D]. Nanjing: Nanjing University of Aeronautics and Astronautics, 2015.

[13] ZHOU Yan, PAN Guoshun, SHI Xiaolei, et al. Atomic step morphology research of LED sapphire substrate polishing surface and its periodicity[J]. Optics and Precision Engineering, 2017，25(1): 100-106.

作者简介: 梁志强(通信作者), 男, 1984 年出生, 副教授, 博士, 博士研 究生导师。主要研究方向为难加工材料切削磨削、微细刀具设计与制造。 E-mail: liangzhiqiang@bit.edu.cn 